\title{
Spin glasses in amorphous and crystalline RE alloys
}

\author{
J. Durand $(*)$ and S. J. Poon $(* *)$ \\ (*) Laboratoire de Structure Electronique des Solides (ERA 100), 4, rue Blaise-Pascal, 67000 Strasbourg, France \\ (**) W. W Hansen Phys. Lab., Stanford University, Stanford, Ca. 94305 U.S.A.
}

\begin{abstract}
Résumé. - Nous présentons un sommaire des principaux résultats expérimentaux obtenus sur les verres de spin dans les systèmes cristallins et amorphes contenant des terres rares. Nous nous intéressons plus spécialement aux propriétés magnétiques moyennes dans les verres de spin métalliques contenant du gadolinium. Les effets du Gd dans la limite diluée $(x \leqslant 1$ at. \%) sont comparés avec ceux mesurés dans les systèmes avec des impuretés $3 \mathrm{~d}$. Le cas des alliages amorphes est discuté. Enfin, nous décrivons en termes d'amas magnétiques les propriétés des verres de spin concentrés $(1 \leqslant x \leqslant 32$ at. $\% \mathrm{Gd})$ dans le système amorphe $\mathrm{La}_{80-x} \mathrm{Gd}_{x} \mathrm{Au}_{20}$.
\end{abstract}

\begin{abstract}
Recent experimental results on spin glasses in crystalline and amorphous RE systems are reviewed. Main emphasis of this paper will be on the bulk magnetic properties of metallic spin glasses containing Gd. The effects of $\mathrm{Gd}$ in the dilute limit $(x \leqslant 1$ at. \%) are compared with those observed in $3 \mathrm{~d}$ impurity systems. The case of amorphous alloys is discussed. Finally, we present a cluster description of magnetic properties in concentrated $\left(1 \leqslant x \leqslant 32\right.$ at. \%) amorphous $\mathrm{La}_{80-x} \mathrm{Gd}_{x} \mathrm{Au}_{20}$ spin glasses.
\end{abstract}

1. Introduction. - Regardless of the (short-or long-range) forces which are responsible for the spinglass phenomena [1], both dilute ( $\sim 1$ at. $\%$, typically) and concentrated spin glasses are phenomenologically characterized by an ordering temperature below which short-range magnetic order [2] and field cooling effects are observed [3-4]. This ordering or freezing temperature $T_{\mathrm{M}}$ is usually defined by a sharp cusp in zero-field ac susceptibility [5]. Considerable effort has developed among theoreticians to explain this cusp in terms of a phase transition [6-7]. Meanwhile, experimentalists have accumulated evidences for the complex nature of the spin-glass ordering process : namely, both the shape and the position of $T_{\mathrm{M}}$ depend on the strength of a dc applied field and on the frequency of an ac field [8-11]. Moreover, specific heat measurements exhibit no singularity at $T_{\mathrm{M}}$; instead, a broad maximum occurs at a temperature $T$ which varies as a function of applied field [12]. In addition, recent neutron scattering experiments were interpreted as indicating a non-unique freezing temperature depending on the momentum of incident neutrons [13]. Finally, the remanence below $T_{\mathrm{M}}$ is time dependent [4]. All these findings do not favor the phase transition picture for the spin-glass freezing. In contrast, they can be interpreted rather easily within a phenomenological model of superparamagnetic clouds [3-4] inspired from Néel's studies on fine grains [14]. Such an approach is very appealing for an experimentalist by providing him with a tool for a quantitative analysis of his data. In particular, the scaling behaviour observed in the dilute limit for $T_{\mathrm{M}}$ and for its frequency dependence [9], for the magnetization [15-16] above
$T_{\mathrm{M}}$ and for the remanence [3] below $T_{\mathrm{M}}$ allows the experimentalist to decide which forces are responsible ( $1 / r^{3}$ interactions). Nevertheless, such a model fails to account for the sharpness of the susceptibility cusp at zero field and low frequency.

In this paper, we review the various manifestations of spin glasses in different systems containing RE. Data on the field-frequency dependence of the freezing temperature or on the relaxation effects in remanence are too scarce yet to be useful for our purpose. In contrast, detailed studies are available concerning the concentration dependence of such quantities as the freezing temperature, the high field magnetization and the remanence in crystalline and amorphous alloys containing $\mathrm{Gd}$ for both dilute and concentrated cases. When analyzing these data, we will assume that $T_{\mathrm{M}}$ or remanence were measured over the same reasonable time scale, so that any concentration dependence study in a given system or any comparison between different spin glasses can still be meaningful.

2. Various spin-glass phenomena in different $\mathbf{R E}$ systems. - In table I are listed the different (pseudo) binary alloys containing RE for which some spinglass properties have been reported. A large number of these alloys and Laves phase compounds were studied within the context of search for coexistence of superconductivity and magnetism. Deviations to the Abrikosov-Gorkov curve, anomalies in the upper critical field and in the specific heat, were attributed to a short-range magnetic order which is now believed to be of spin-glass type. These properties together with 
Table I. - Spin-glass phenomena in RE systems.

\begin{tabular}{|c|c|c|c|}
\hline & Matrixes & Impurities & Spin-glass phenomena \\
\hline 1. Alloys & $\begin{array}{l}\mathrm{Sc} \\
\mathrm{Y} \\
\mathrm{La} \\
\mathrm{Pr} \\
\mathrm{La}_{98} \mathrm{Lu}_{2} \\
\mathrm{Th}\end{array}$ & $\begin{array}{l}\text { Gd, Ho, Er, Dy } \\
\text { Gd, Tb, Dy } \\
\text { Gd, Eu } \\
\mathrm{Nd}, \mathrm{Tb} \\
\mathrm{Tb} \\
\mathrm{Er}\end{array}$ & $\begin{array}{l}T_{\mathrm{M}}\left(^{*}\right)[18], C_{\mathrm{v}}(*)[19], \mathrm{ME}\left(^{*}\right)[20], \mathrm{N}\left(^{*}\right)[20] \\
T_{\mathrm{M}}[18,21], C_{\mathrm{v}}[19,22] \\
\operatorname{sc}\left(^{*}\right)[23], \mathrm{TM}[23], C_{\mathrm{v}}[23], \mathrm{ME}[24] \\
T_{\mathrm{M}}[18] \\
\mathrm{sc}, T_{\mathrm{M}}[25] \\
\mathrm{sc}, T_{\mathrm{M}}[26]\end{array}$ \\
\hline 2. Compounds & $\begin{array}{l}\mathrm{YOs}_{2} \\
\mathrm{LaAl}_{2} \\
\mathrm{LaB}_{6} \\
\mathrm{La}_{3} \mathrm{Al} \\
\mathrm{La}_{3} \mathrm{In} \\
\mathrm{LaRu}_{2} \\
\mathrm{LaSn}_{3} \\
\mathrm{CeRu}_{2} \\
\mathrm{ThRu}_{2} \\
\mathrm{SnMo}_{6} \mathrm{~S}_{8} \\
\mathrm{Bi}_{3} \mathrm{Sr}\end{array}$ & $\begin{array}{l}\mathrm{Gd} \\
\mathrm{Gd}, \mathrm{Ce} \\
\mathrm{Gd}, \mathrm{Ce} \\
\mathrm{Gd} \\
\mathrm{Gd}, \mathrm{Ce} \\
\mathrm{Gd}, \mathrm{Ce}, \mathrm{Pr} \\
\mathrm{Gd}, \mathrm{Tb} \\
\mathrm{Pr}, \mathrm{Gd}, \mathrm{Tb}, \mathrm{Dy}, \mathrm{Ho} \\
\mathrm{Gd} \\
\mathrm{Eu} \\
\mathrm{Eu}\end{array}$ & $\begin{array}{l}\text { sc, } C_{\mathrm{v}}[27] \\
\text { sc [28], } T_{\mathrm{M}}[9,29-31], C_{\mathrm{v}}[12,32-33], \text { ESR [34], } \\
\text { RMN }[35] \\
T_{\mathrm{M}}, M_{\mathrm{rs}}(*)[36,37] \\
\text { sc, } T_{\mathrm{M}}[38] \\
\text { sc, } T_{\mathrm{M}}[39,40] \\
\text { sc }[41,42] \\
\text { sc, } T_{\mathrm{M}}[43], \mathrm{N}[44] \\
\text { sc }[45], C_{\mathrm{v}}[46], \mathrm{ME}[47-48], T_{\mathrm{M}}[49], \mathrm{N}[50] \\
\mathrm{sc}, T_{\mathrm{M}}[49] \\
\mathrm{sc}, \mathrm{N}[51] \\
\mathrm{sc}, T_{\mathrm{M}}, C_{\mathrm{v}}[52]\end{array}$ \\
\hline 3. Amorphous & $\begin{array}{l}\mathrm{Cu} \\
\mathrm{Al} \\
\mathrm{CuZr} \\
\mathrm{La} \\
\mathrm{La}_{80} \mathrm{Au}_{20}\end{array}$ & $\begin{array}{l}\text { Gd, Dy } \\
\text { Gd } \\
\text { Gd, Tb } \\
\text { Gd } \\
\text { Gd }\end{array}$ & $\begin{array}{l}T_{\mathrm{M}}[53], C_{\mathrm{v}}[54], \operatorname{ESR}[55] \\
T_{\mathrm{M}}[53] \\
{[56]} \\
{[57]} \\
\operatorname{sc}[58], T_{\mathrm{M}}[59-60], M_{\mathrm{rs}}[61]\end{array}$ \\
\hline 4. Insulators & $\mathrm{SrS}$ & $\mathrm{Eu}$ & $T_{\mathrm{M}}[10,62], \mathrm{ME}[10], M_{\mathrm{rs}}[4], \mathrm{N}[10]$ \\
\hline
\end{tabular}

$\left(^{*}\right) T_{\mathrm{M}}=$ zero-field susceptibility; $C_{\mathrm{v}}=$ specific heat; $\mathrm{ME}=$ Mössbauer effect $; \mathrm{N}=$ neutron diffraction ; sc = superconducting properties; $M_{\mathrm{rs}}=$ saturated remanence.

the results of Mössbauer spectroscopy and neutron diffraction studies in these superconducting spin glasses have been recently reviewed [17].

As can be seen from table I, there exists a large variety of spin glasses containing RE. We should add to the list the spin-glass-like regimes obtained at low temperature in some compounds like $\mathrm{GdCo}_{2}$ after $\mathrm{H}_{2}$ absorption [63]. On the other hand, Chevrel phases like $\operatorname{Re}_{x} \mathrm{Mo}_{6} \mathrm{~S}_{8}$ could be good candidates for a new class of spin glasses [64]. Not included in the list are concentrated amorphous alloys such as REAg [65], where, despite of large hysteresis loops and strong after effects, there exists a long-range magnetic order. This kind of amorphous RE alloys was reviewed recently by Coey [66] and Cochrane et al. [67].

It would be of interest to compare the magnetic properties of Gd spin glasses with those of spin glasses containing other RE elements. Recent calculations [68] investigated the possibility for a local uniaxial anisotropy to induce a spin-glass-like behaviour. Unfortunately, experimental results available so far do not allow to determine unambiguously the effects of the magnetic interactions and those due to crystal field effects. For example, the ac susceptibility maximum is broad in ScTb alloys and it becomes sharper when a dc field is applied. This behaviour, opposite to that observed in $\mathrm{ScGd}$, was attributed to the single ion anisotropy [18]. But this effect does not seem to be the general case, since a sharp cusp was observed in a $\mathrm{Y}_{98} \mathrm{~Tb}_{2}$ alloy [22]. On the other hand, the critical concentration for the onset of long-range magnetic order for $\mathrm{Tb}$ in $\mathrm{Y}$ is twice that for $\mathrm{Gd}$ in $\mathrm{Y}$ (5 and 2.6 at. $\%$, respectively) [18]. In contrast, the critical concentration is about the same for $\mathrm{Tb}$ and $\mathrm{Gd}$ in a $\mathrm{Sc}$ matrix (22 and 24 at. \%, respectively) [18]. More detailed investigations are needed on non- $S$ state spin-glass systems before one can make a meaningful comparison with the simple case of spin-glasses containing Gd.

\section{Crystalline and amorphous Gd spin glasses in the} dilute limit. - 3.1 CRYSTALline Gd AND 3d SPIN GLASSES. - Influence of RKKY interactions. Are listed in table II the experimental values for $T_{\mathrm{M}} / x, V_{0}$ and $S$ obtained in the dilute limit ( 1 at. $\%$ ) for some typical $\mathrm{Gd}$ and $3 \mathrm{~d}$ spin-glasses. The strength of the RKKY interaction $V_{0}$ was determined from approach to saturation [77]. From table II, it appears at first sight that $T_{\mathrm{M}} / x$ scales roughly with $V_{0}$. This correlation between $T_{M} / x$ and $V_{0}$ can be expressed in a very naive way as follows. Our starting point is 
Table II. - Values of spin-glass parameters in $4 \mathrm{f}$ and $3 \mathrm{~d}$ systems.

\begin{tabular}{llll}
\multicolumn{1}{c}{ Alloy } & \multicolumn{1}{c}{$T_{\mathrm{M}} / x(\mathrm{~K} / \mathrm{at} . \%)$} & \multicolumn{1}{c}{$V_{0}\left(10^{-37} \mathrm{erg} \mathrm{cm}^{3}\right)$} & \\
$\mathrm{ScGd}$ & $0.55[18]$ & & \\
$\overline{\mathrm{YG}}$ & $2.30[18]$ & & \\
$\overline{\mathrm{LaGd}}$ & $0.55[23]$ & & $3.5[30]$ \\
$\overline{\mathrm{La}}_{3-x} \mathrm{Gd}_{x} \mathrm{In}$ & $0.60[39]$ & & $3.5[36]$ \\
$\mathrm{Ce}_{1-x} \mathrm{Gd}_{x} \mathrm{Ru}_{2}$ & $0.70[48]$ & $0.24[30]$ & $3.5[60,69]$ \\
$\mathrm{La}_{1-x} \mathrm{Gd}_{x} \mathrm{Al}_{2}$ & $0.26[30]$ & $0.24[36]$ & $1.5[70]$ \\
$\mathrm{La}_{1-x} \mathrm{Gd}_{x} \mathrm{~B}_{6}$ & $0.09[36]$ & $0.3[60,69]$ & $0.9-1.2[72-73]$ \\
a) & $0.50[59]$ & $11[70]$ & $2[74]$ \\
$\mathrm{La}_{80-x} \mathrm{Gd}_{x} \mathrm{Au}_{20}$ & $12[4]$ & $2.4-3.8[72-73]$ & $2[74]$ \\
$\overline{\mathrm{AuFe}}$ & $0.55[71]$ & $7.5[74]$ & $2.2[74]$ \\
$\overline{\mathrm{MoFe}}$ & $17[4]$ & $3.5[74]$ & $2[76]$ \\
$\overline{\mathrm{CuMn}}$ & $(*)(4.5)[75]$ & $2.4[74]$ &
\end{tabular}

(*) Values given for $T_{\mathrm{M}} / x$ in AgMn and AuMn alloys are only an estimate. The dilute limit for which $T_{\mathrm{M}}$ should scale with $x$ has not been reached [75].

the simple derivation given by Sherrington [78] for the order parameter of the Edwards-Anderson model [79]. Sherrington obtains for $T_{M}$ an expression which is in fairly good agreement with those deduced from more rigorous treatments $[80-81]$ :

$$
T_{\mathrm{M}}=S(S+1) /(3 \sqrt{3}) \cdot k_{\mathrm{B}}^{-1}\left(\overline{\sum_{\mathrm{j}} J_{\mathrm{ij}}^{2}}\right)^{1 / 2}
$$

where the average $\left(\overline{\sum_{j} J_{i j}^{2}}\right)^{1 / 2}$ is related to the halfwidth of the molecular field distribution $\Delta$ by

$$
\Delta=S\left(\overline{\sum_{\mathrm{j}} J_{\mathrm{ij}}^{2}}\right)^{1 / 2}
$$

In metallic systems with infinite mean-free-path, the $J_{i j}$ 's can be expressed by the RKKY function in its asymptotic form. By following the molecular field approach of Souletie [82] inspired from Klein and Brout [83], we can calculate $\triangle$ :

$$
\Delta=11.85 Q x S V_{0} d^{-3}
$$

where $d$ is the lattice constant of a cubic cell, and $Q$ equals to $1 / 4,1 / 2$ and 1 for the s.c., b.c.c. and f.c.c. structures, respectively. Substituting (2) in (1) yields an explicit expression for the freezing temperature :

$$
T_{\mathrm{M}}=2.28 Q k_{\mathrm{B}}^{-1} x S(S+1) V_{0} d^{-3} .
$$

This expression derived for an Heisenberg model should be multiplied by $\sqrt{3}$ for an Ising model [78].

The validity of equation (3) is checked on figure 1 by plotting the experimental values for $T_{\mathrm{M}} / x$ normalized as $\left(T_{\mathrm{M}} d^{3} k_{\mathrm{B}}\right) /(x Q S(S+1))$ as a function of $V_{0}$. There is no doubt that equation (3) accounts for the general trend of experimental data for both $4 \mathrm{f}$ and $3 \mathrm{~d}$ spin-glasses. That the data are better fitted to a

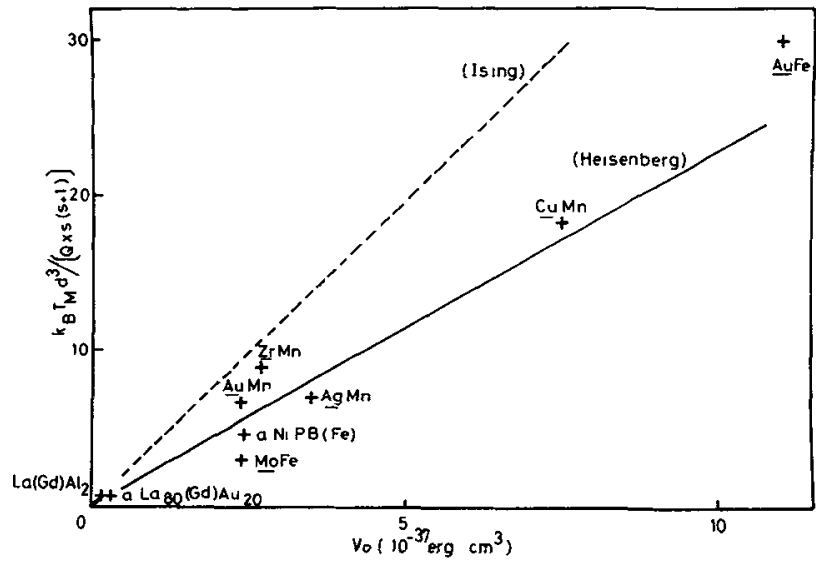

Fig. 1. - Experimental values for $T_{\mathrm{M}}$ in $4 \mathrm{f}$ and $3 \mathrm{~d}$ spin glasses (see references on table II) normalized as $\left(k_{\mathrm{B}} T_{\mathrm{M}} d^{3}\right) / x Q S(S+1)$ versus strength $V_{0}$ of the RKKY interaction. Theoretical predictions from equation (3) are indicated for an Heisenberg model (full line) and for an Ising model (dotted line).

Heisenberg model than to an Ising model might be fortuitous, considering the very crude character of our derivation.

3.2 AMORPHOUS AND CRYSTALLINE Gd SPINGLASSES. - It can be seen in table II that the values for $V_{0}$ as determined from approach to saturation are practically identical in amorphous and crystalline Gd spin glasses. In addition, the values for $T_{M} / x$ are rather close once the Gd concentration is taken with respect to the whole matrix. From $V_{0}$, we can deduce a value for $J_{\text {sf }}$ which is in good agreement with that obtained from the depression of the superconducting transition in the dilute limit [58]. Such an agreement asserts that the $1 / H$ contribution in approach to saturation is mainly RKKY in nature. On the 
other hand, the initial susceptibility and the magnetization [60] for dilute $(x<1$ at. $\%)$ amorphous $\mathrm{La}_{80-x} \mathrm{Gd}_{x} \mathrm{Au}_{20}$ were found to follow the SouletieTournier scaling laws [16]. This indicates that the RKKY interaction remains basically in its $1 / r^{3}$ asymptotic form within this amorphous matrix. A $1 / r^{3}$ dipolar interaction is not likely to be predominant for the aforementioned reasons.

This result is rather unexpected. From resistivity measurements, the electronic mean-free-path does not exceed $10 \AA$ in an amorphous alloy. Thus, according to the exponential damping factor of de Gennes [84] and others [85], one should expect a severe modification of the magnetic superconducting properties in an amorphous medium. Our result seems to contradict previous experimental work on the mean-free-path effects in dilute crystalline spin glasses [82, 86-88]. This problem deserves further experimental and theoretical investigations.

4. From dilute to concentrated spin glasses in amorphous $\mathrm{La}_{80-x} \mathbf{G d}_{x} \mathbf{A u _ { 2 0 }}$ alloys. - When the $\mathrm{Gd}$ concentration exceeds 1 at. $\%$ in amorphous $\mathrm{La}_{80-x} \mathrm{Gd}_{x} \mathrm{Au}_{20}$ alloys, the reduced magnetization $M / x$ does not scale anymore with the reduced parameters $H / x$ and $T / x$. On the other hand. $T_{\mathrm{M}}$ still varies linearly with $x$ up to $x=12$, and the departure from linearity is rather small up to $x \simeq 30$ [59]. Moreover, the reduced saturated remanence $M_{\mathrm{rs}}{ }^{\prime} x$ was found to scale fairly well with $T / x$ for $G d$ concentrations as large as 32 at. \% [61]. We tried to resolve this apparent contradiction within a model of small interacting clusters [69] which may shed some light on the mechanisms involved in the freezing process of concentrated spin-glasses.

We assume that for concentrations above 1 at. $\%$ some ferromagnetic clusters are built up whose spin is $S^{*}$ and concentration $x^{*}$. These ferromagnetic clusters do not overlap, so that $x^{*} S^{*}=x S$. The Blandin-Souletie-Tournier scaling argument [15-16] can be extended to magnetic clusters with $x^{*} R_{\mu \nu}^{3}$ as a new invariance, $R_{\mu \nu}$ being the average distance between clusters. Thus, the scaling law for magnetization modified for clusters reads :

$$
M / x=f\left(H / x^{*}, T / x\right) .
$$

A nice scaling behaviour of this type was found in amorphous $\mathrm{La}_{80-x} \mathrm{Gd}_{x} \mathrm{Au}_{20}$ by determining $x^{*}$ graphically for each value of $x$ up to $x=32$ at. $\%$ (figure 2). Above 32 at. $\%$, the scaling behaviour cannot be recovered any more by simple adjustment of one reduced parameter (figure $2 b$ ). This indicates that our simple model fails to describe the magnetization as the clusters start to overlap. Knowing $x^{*}$ allows us to determine the cluster size from $S^{*} / S=x / x^{*}$. This size can be correlated with the mean environment of a Gd atom in our amorphous matrix. From linear extrapolation of structural studies [89], the Gd-Gd coordination number $\mathrm{CN}$ is $x / 10$, so that $\mathrm{CN}$ is 8 in $\mathrm{Gd}_{80} \mathrm{Au}_{20}$. It can be seen on figure 3 that up to $x=24$ at. $\%$ the cluster size $S^{*} / S$ is fairly well determined by a given $\mathrm{Gd}$ atom surrounded by its $\mathrm{Gd}$ first neighbours, i.e. $Z=1+(x / 10)$. Above 24 at. $\%$, $S^{*} / S$ is considerably larger than $Z$, which manifests the emergence of cluster percolation.

The strength $V_{0}^{*}$ of the effective intercluster interaction can be determined from the Larkin-Khmel'nitskii method modified for clusters. $V_{0}^{*}$ was found to vary in such a way that $V_{0}^{*} S^{*}=V_{0} S$ (figure 3). We believe that this interaction is mainly of the RKKY type, since for $x<32$, the ratio $M_{\mathrm{rs}}(0) / M_{\infty}(0)$ is equal to about $5 \times 10^{-2}$, which provides a measure of the amplitude of the RKKY exchange over the dipolar coupling [4].

Back to the concentration dependence of $T_{\mathrm{M}}$, our model can explain that $T_{\mathrm{M}}$ remains linear with $x$ in concentrated spin glasses, since equation (3) remains unchanged as long as the relations $x^{*} S^{*}=x S$ and $\left(S^{*}+1\right) V_{0}^{*} \simeq(S+1) V_{0}$ are satisfied. Similarly, it can be shown that the scaling behaviour for the remanence is observed provided that $T_{\mathrm{M}}$ versus $x$ does not depart too strongly from linearity.

According to this analysis, the freezing temperature of metallic spin glasses would be determined mainly
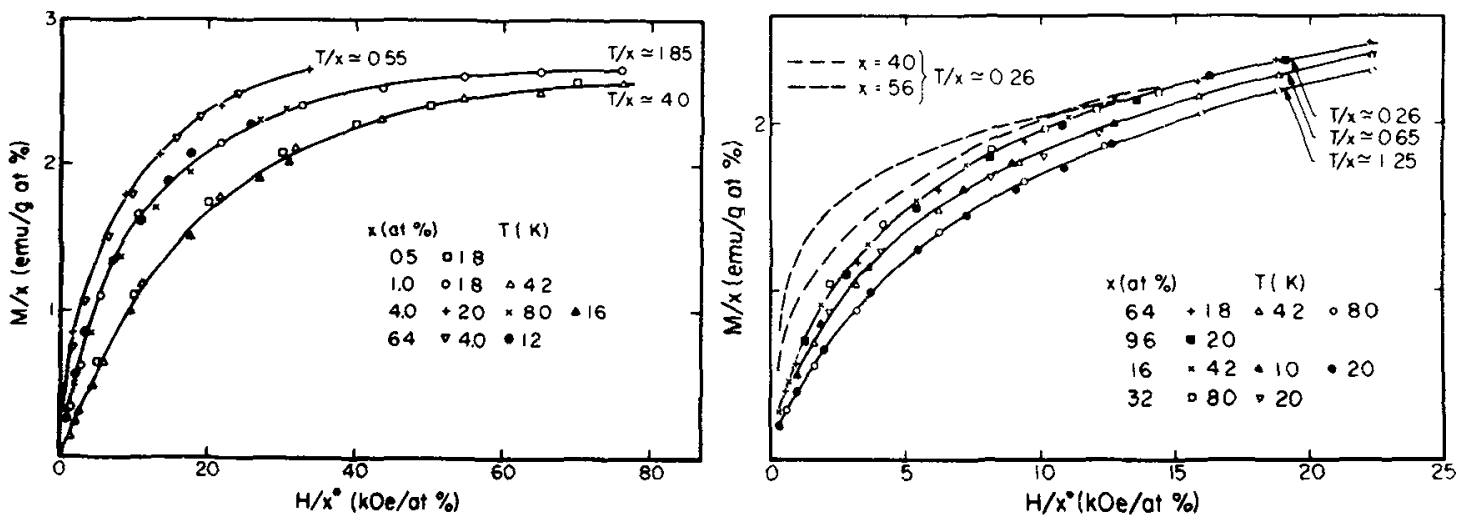

Fig. 2. - Reduced magnetization $M / x$ of $\mathrm{La}_{80-x} \mathrm{Gd}_{x} \mathrm{Au}_{20}$ amorphous alloys as a function of reduced magnetic field for clusters $H / x^{*}$ at different reduced temperatures $(a)$ for $0.5 \leqslant x \leqslant 6.4$ alloys, $(b)$ for $6.4 \leqslant x \leqslant 32$ alloys. 


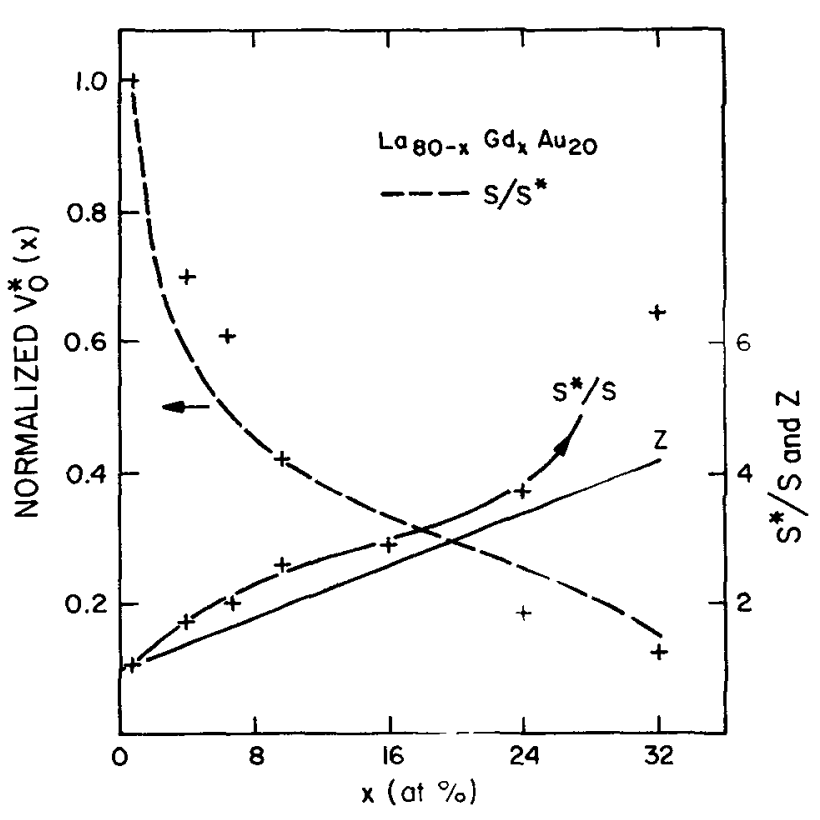

Fig. 3. - Cluster size $S * / S$ and normalized effective intercluster coupling $V_{0}^{*} / V_{0}$ versus Gd content. The variation of $S^{*} / S$ is compared with that of $Z$ (see text). The values for $V_{0}^{*} / V$ (approach to saturation) are compared with the values for $S / S^{*}$ (from scaling laws modified for clusters).

by the strength of the RKKY interaction between isolated spins within the dilute limit. and between small clusters $\left(S^{*} / S=3.75\right.$ for $\left.x=24\right)$ in concentrated spin-glasses. These clusters behave at high field like single spin entities of concentration $x^{*}=x / Z$, having a spin $S^{*}=S Z$; they are coupled by a $1 / r^{3}$ interaction mainly of RKKY type with an amplitude $V_{0}^{*}=V / Z$. Let us note that, as in the Soukoulis and Levin model [90], an average size cluster has been used instead of a distribution of cluster size [4]. On the other hand, our small ferromagnetic clusters resemble the superspins in the Monte Carlo simulations of Binder [7]. The remanence phenomena would imply a more complex picture of larger clouds made of 40-50 spins or superspins for our alloys, the size of these clouds being governed by an interplay of RKKY and dipolar interactions between spins or small clusters [4].

5. Conclusion. - Due to highly localized $4 \mathrm{f}$ electrons, large $\mathrm{Gd}$ moment, negligible crystal-field effects, it appears that the alloys containing $\mathrm{Gd}$ provide a simple case to illustrate the importance of the RKKY exchange on spin-glass phenomena and to analyze the magnetic properties of concentrated spin glasses. Much could be learned also from detailed concentration dependence investigations on metallic and insulating spin glasses containing RE elements in a non-S ionic state. This latter area remains almost unexplored.

\section{References}

[1] For experimental aspects of spin glasses, see the reviews of BECK, P. A., Met. Trans. 2 (1971) 2015 ;

MYdosf, J. A., J. Mag. Mag. Mat. 7 (1978) 237;

Souletie, J., J. Physique Colloq. 39 (1978) C 2-3.

[2] Window, B., J. Mag. Mag. Mat. 1 (1975) 167.

[3] Tholence, J. L. and Tournier, R., J. Physique Colloq. 35 (1974) C 4-229.

[4] Holtzberg, F., Tholence, J. L. and Tournier, R., in Amorphous Magnetism II, edit. Levy and Hasegawa (Plenum Press, New York) 1977, p. 155.

[5] Cannella. V., Mydosh, J. A. and Budnick, J. I., J. Appl. Phys. 42 (1971) 1689.

[6] For recent theoretical developments on spin glasses, see : Anderson, P. W., J. Appl. Phys. 49 (1978) 1599 ;

FISCHER, K. H., Physica 86-88B (1977) 813;

KINZEL, W. and FISCHER, K. H., J. Phys. C 11 (1978) 2115.

[7] BINDER, K., Z. Phys. B 26 (1977) 339; and in Festkörperprobleme XVII, edit. Treusch (Vieweg, Braunschweig) 1977, p. 55; J. Physique Colloq. 39 (1978) C 6-1527.

[8] Mukhopadhyay, A. K., Shull, R. D. and BeCK, P. A., J. Less-Common Met. 43 (1975) 69.

[9] V. LöhneYsen, H., Tholence, J. L. and TOURNier, R., in LT 15 Conf. Proc., J. Physique Colloq. 39 (1978) C 6-922.

[10] Maletta, H. and FelsCh, W., in LT 15 Conf. Proc., J. Physique Colloq. 39 (1978) C 6-931;

Maletta, H. and Convert, P., Phys. Rev. Lett. 42 (1979) 108.

[11] Tholence, J. L., Holtzberg, F., Godfrin, H., V. LöhneySEN, H. and TOUR NIER, R., in LT 15 Conf. Proc., J. Physique Colloq. 39 (1978) C 6-928.
[12] Bredl, C. D., Steglich, F., V. Löhneysen, H. and Matho, K., in LT 15 Conf. Proc., J. Physique Colloq. 39 (1978) C 6-925.

[13] Murani, A. P., J. Appl. Phys. 49 (1978) 1604 ; J. Physique Collog. 39 (1978) C 6-1499.

[14] Neel, L., Adv. Phys. 4 (1955) 191; J. Phys. Soc. Japan 17 Sup. B-I (1962) 676.

[15] Blandin, A., Thèse, Paris (1961).

[16] Souletie, J. and Tournier, R., J. Low Temp. Phys. 1 (1969) 95.

[17] Maple, M. B., Appl. Phys. 9 (1976) 179;

Roth, S., Appl. Phys. 15 (1978) 1.

[18] Sarkissian, B. V. B. and Coles, B. R., Commun. Phy's. 1 (1976) 17 ;

Sarkissian, B. V. B., J. Phys. F 7 (1977) L 139 ; Physica 8688B (1977) 865; and this conference ( $J$. Physique Colloq. 40 (1979) C 5).

[19] Isaacs, L. L., Phys. Rev. B 8 (1973) 3301 ; AIP Conf. Proc. 18 (1974) 312 ;

Levesque, B., Caudron, R. and Costa, P., this conference (J. Physique Colloq. 40 (1979) C 5).

[20] Abbundi, R., Rhyne, J. J., Sweger, D. M. and Segnan. R., AIP Conf. Proc. 29 (1976) 352; Phys. Rev. B 18 (1978) 3313 , and references therein.

[21] Nagasawa, H. and Sugawara, T., J. Phys. Soc. Japan 23 (1967) 711.

[22] Wenger, L. E., J. Appl. Phys. 49 (1978) 1630.

[23] Hein, R. A., Falge, R. L., Matthias, B. T. and Corenzwit, C., Phys. Rev. Lett. 2 (1959) 500 ;

Phillips, N. E. and Matthias, B. T., Phys. Rev. 121 (1961) 105. 
[24] Steiner, P., Gumprecht, D. and Hüfner, S., Phys. Rev. Lett. 30 (1973) 1132 ;

Gumprecht, D., Steiner, P. and HüfNer, S., Phys. Lett. 48 (1974) 269.

[25] Williams, L. J., Decker, W. R. and Finnemore, D. K., Phys. Rev. B 2 (1970) 1287.

[26] Gulrtin, R. P. and Parks, R. D., Solid State Commun. 7 (1969) 59.

[27] Suhl, H., Matthias, B. T. and Corenzwit, E., J. Phys. Chem. Solids 11 (1959) 346.

[28] Maple, M. B., Phys. Lett. 26A (1968) 513.

[29] Bennett, M. H. and Coles, B. R., Physica 86-88B (1977) 884.

[30] V. LöhneYSEN, H., ThOlENCE, J. L. and STEGLiCh, F., Z. Phys. B 29 (1978) 319.

[31] Felsch, W., WinZer, K. and V. MinNigerode, G., Z. Phys, $B 21$ (1975) 151.

[32] StEGLICH, F, in Festkörperprobleme XVII, edit. Treusch (Vieweg, Braunschweig) 1977, p. 319.

[33] Trainor, R. J. and McCollum, D. C., Phys. Rev. B 9 (1974) $2145 ; 11$ (1975) 3581.

[34] Davidov, D., Chelkowski, A., Rettori, C., Orbach, R. and Maple, B. R., Phys. Rev. B 7 (1973) 1029.

[35] Maclaughlin, D. E., J. Low Temp. Phys. 26 (1977) 111.

[36] FelsCh, W., J. Mag. Mag. Mat. 6 (1977) 103 ; Z. Phys. B 29 (1978) 203.

[37] FelsCh, W., Z. Phys. B 29 (1978) 211.

[38] Kuwasara, Y., Sekizawa, K., Usui, N. and Yasukochi. K., J. Phys. Soc. Japan 27 (1969) 590.

[39] Guertin, R. P., Crow, J. E. and Parks, R. D., Phys. Rev. Lett. 16 (1966) 1095.

[40] Prejean, J. J. and Souletie, J., in Int. Conf. Mag. Proc. (1974, Publishing House Nauka) vol. 4, p. 437.

[41] Jones, H., Fischer, $\emptyset$., Bongi, G. and Treyvaud, D., Solid State Commun. 10 (1972) 927 ;

Hillendrand, D. and WhilHELM, M., Phys. Lett. 40A (1972) 387.

[42] Jones, T. E., Chock, E. P. and Chaikin, P. M., Bull. Am. Phys. Soc. 22 (1977) 339.

[43] Toxen, A. M., Kwok, P. C. and Gambino, R. J., Phys. Rev. Lett. 21 (1968) 792 ;

Schmid, W. and Umlauf, E., Commun. Phys. 1 (1976) 67.

[44] Hoenig, H. E. and V. Blanckenhagen, P., in Crystal-Field Effects in Metals and Alloys, edit. Furrer (Plenum Press, New York) 1977, p. 128.

[45] Matthias, B. T., SumL, H. and Corenzwit, E., Phys. Rev. Lett. 1 (1958) 449 ;

Wilhelm, M. and Hillendrand, B., J. Phys. Chem. Solids 31 (1970) 559 ; Physica 55 (1971) 608 ;

Peter, M. et al., Helv. Phys. Acta 44 (1971) 345.

[46] Rupp, G., J. Phys. F 3 (1973) 1403.

[47] TAYLOR, R. D. et al., in LT 13 Conf. Proc., edit. K. D. Timmerhaus et al. (Plenum Press, New York) 1974, p. 605.

[48] Ruebendauer, K., Fink, J., Schmidt, H., Czjzek, G. and Tomala, K., Phys. Status Solidi (b) 84 (1977) 611.

[49] Davidov, D., Baberschke, K., Mydosh, J. A. and NieuWenHUYs, G. J., J. Phys. F 7 (1977) L 47.

[50] Roth, S., IBeL, K. and Just, W., J. Phys. C 6 (1973) 3465.

[51] Bolz, J., Crecelrus, G., Maletta, H. and Pobell, F., J. Low Temp. Phys. 28 (1977) 61.

[52] KempF, B., Elschner, B., Spttzli, P. and Fischer, ф., Phys. Rev. B 17 (1978) 2163.

[53] McGuire, T. R., Mizoguchi, T., Gambino, R. J. and KiRKPATRICK, S., J. Appl. Phys. 49 (1978) 1689 ; Phys. Rev. Lett. 38 (1977) 89.

[54] Coey, J. M. D., V. Molnar, S. and Gambino, R. J., Solid State Commun. 24 (1977) 167, J. Physique Lett. 39 (1978) L-327.

[55] Malozemoff, A. P. and Jamet, J. P., Phys. Rev. Lett. 39 (1977) 1293 and Phys. Rev. B 18 (1978) 75.

[56] Gruzalski, G. R., Weymouth, J. W., Sellmyer, D. J. and
GIESsEN, B. C., in Amorphous Magnetism II, edit. R. A. Levy and R. Hasegawa (Plenum Press, New York) 1977, p. 235; in Toronto Conf. Proc. on Transit. Metals Inst. Phys. Conf. Ser. 39 (1978) 445.

[57] Korn, D., Z. Phys. 187 (1965) 463.

[58] Poon, S. J. and Durand, J., Solid State Commun. 21 (1977) 999.

[59] Poon, S. J. and Durand, J., in reference [4], p. 245.

[60] Poon, S. J. and Durand, J., Solid State Commun. 21 (1977) 793.

[61] Poon, S. J. and Durand, J., Commun. Phys. 2 (1977) 87

[62] Maletta, H. and Crecelius, G., J. Physique Colloq. 37 (1976) C 6-645.

[63] Buschow, K. H. J., J. Less-Common Met. 51 (1977) 173 ;

Malik, S. K. and Wallace, W. E., Solid State Commun. 24 (1977) 417.

[64] Fischer, $\emptyset .$, Appl. Phys. 16 (1978) 1.

[65] Boucher, B., IEEE Trans. Mag. 13 (1977) 1601; and this conference (J. Physique Colloq. 40 (1979) C5).

[66] CoEY, J. M. D., J. Appl. Phys. 49 (1978) 1646.

[67] Cochrane, R. W., Harris, R. and Zuckermane, M. J., Phys. Rep. 48 (1978) 1.

[68] ZuckermanN, M. J., this conference (J. Physique Colloq. 40 (1979) C 5).

[69] Poon, S. J. and Durand, J., Phys. Rev. B 18 (1978) 6253.

[70] SMith, F. W. and Liu, J. C., Solid State Commun. 26 (1978) 91.

[71] Caudron, R. et al., Physica 86-88B (1977) 833.

[72] Amamou, A. et al, J. Phys. F 6 (1976) 2371.

[73] Smith, F. W. and Sarachik, M. P., Phys. Rev. B 16 (1977) 4142.

[74] SMITH, F. W., Phys. Rev. B 13 (1976) 2976 ; B 14 (1976) 241.

[75] Cannella, V. and Mydosh, J. A., AIP Conf. Proc. 18 (1974) 651.

[76] Jones, H. C. et al., Phys. Rev. B 16 (1977) 1177.

[77] Larkin, A. I. and Krimgl'Nitski, D. E., Sov. Phys. JETP 31 (1970) 958 ;

Larkin, A. I., Mel'nikov, V. I. and KhMEL'NITSKII, D. E., Sov. Phys. JETP 33 (1971) 458;

MATHO, K., Physica 86-88B (1977) 854.

[78] Sherrington, D., J. Phys. C 8 (1975) L 208.

[79] Edwards, S. F. and Anderson, P. W., J. Phys. F 5 (1975) 965.

[80] Sherrington, D. and KirkPatrick, S., Phys. Rev. Lett. 35 (1975) 1792.

[81] Fischer, K. H., Phys. Rev. Lett. 34 (1975) 1348 ; Solid State Commun. 18 (1976) 1515.

[82] Souletie, J., Thèse, Grenoble (1968);

BetHoux, O. et al., in LT 10 Conf. Proc., edit. Borovik-Romanov et Tulin (Moscow Univ.) 1967, vol. 4, p. 290 ;

SOuletie, J., Tholence, J. L. and Tournier, R., in $L T 11$ Conf. Proc., edit. Allen et al. (St Andrews Univ., Scotland) 1968 vol. 2, p. 1263.

[83] Klein, M. W. and Brout, B., Phys. Rev. 132 (1963) 2412; KleIN, M. W., Phys. Rev. 136 (1964) 1156 ; 173 (1968) 552; 188 (1969) 933.

[84] De Gennes, P. G., J. Physique Radium 23 (1962) 630.

[85] Madhukar, A., J. Physique Colloq. 35 (1974) C 4-295; Kaneyoshi, T., J. Phys. F 5 (1975) 1014.

[86] Hegger, A. J., KLien, A. P. and Tu, P., Phys. Rev. Lett. 17 (1966) 803 ;

WALSTEDt, R. E. and WALKer, L. R., Phys. Rev. B 9 (1974) 4857.

[87] Buchmann, R., Falke, H. P., Jablonski, H. P. and WasserMann, E. F., Physica 86-88B (1977) 835; Phys. Rev. B 17 (1978) 4315.

[88] Larsen, U., Solid State Commun. 22 (1977) 311.

[89] Logan, J., Scr. Metall. 9 (1975) 379.

[90] Soukoulis, C. M. and Levin, K., Phys. Rev. Lett. 39 (1977) 581. 